(C2017, Elsevier. Licensed under the Creative Commons Attribution-NonCommercial-NoDerivatives

4.0

International

http://creativecommons.org/about/downloads

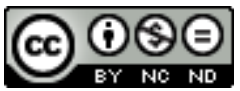




\title{
Contact angle hysteresis analysis on superhydrophobic surface based on the design of channel and pillar models
}

\author{
Zhenyu Shi ${ }^{\mathrm{a}, \mathrm{b}}$, Xianzhi Zhang ${ }^{\mathrm{c}}$ \\ ${ }^{a}$ Key Laboratory of High Efficiency and Clean Mechanical Manufacture \\ (Shandong University), Ministry of Education, Jinan, P. R. China) \\ ${ }^{\mathrm{b}}$ School of Mechanical Engineering, Shandong University, Jinan, P. R. China \\ ${ }^{\mathrm{c}}$ School of Mechanical and Aerospace Engineering, Kingston University, London, UK \\ *Corresponding author. Tel.: +442084176635, Fax.: +442084176635, E-mail: X.Zhang@ @ingston.ac.uk
}

\begin{abstract}
Contact Angle Hysteresis $(\mathrm{CAH})$ is critical to the hydrophobicity of a surface, which describes the dynamic characteristic of droplets. In this paper, two different micro-structured surfaces respectively with micro-channel and micro-pillar structures (20 samples for each structure, with a range of channel and pillar widths between 25 to $250 \mu \mathrm{m}$ ) were fabricated by mechanical micro-milling process to investigate the effect of structural parameters on hydrophobicity of surfaces. It was found that the solid fraction plays a decisive role for a surface in the transition from being hydrophilic to hydrophobic. Quantitative interpretation was conducted and a dynamic methodology was established based on the physical nature of the controllable motion of a droplet. The five key states of a droplet including the initial, pre-forward, forward, pre-backward and backward were the main focus of this research. The prediction results based on the established model showed good consistency with experiments. The proposed model can estimate the advancing and receding angles very well. The outcome of this research will lead to new methodologies for preparing hydrophobic surfaces with micro-machining technology and play an important theoretical guiding role in fabrication of superhydrophobic surfaces.
\end{abstract}

KEYWORDS : Hydrophobic surface, Contact angle hysteresis, Advancing angle, Receding angle, Micro-milling. 


\section{Introduction}

For rough or chemically heterogeneous surfaces, metastable phenomenon for the wetting system may occur. The variations of the contact angle are within a range which corresponds to a series of dynamic contact angles [1-2]. The maximum value of contact angle before the drop advances is called advancing angle, and the minimum value of the contact angle before the drop retracts is called receding angle [3-4]. Contact angle hysteresis $(\mathrm{CAH})$ is considered to be the difference between the advancing angle and the receding angle. The $\mathrm{CAH}$ reveals the contact angle spectrum, within which drops are inhibited of motions. The advancing angle reflects the property of hydrophobicity, while the receding angle indicates the property of hydrophilicity [5].

The use of superhydrophobic surfaces is essentially self-cleaning in nature [6]. Most reported studies have applied superhydrophobic surfaces to increase the extent of hysteresis on neighboring hydrophobic or hydrophilic regions [7]. In recent years, with the rapid development of science and technology, ultra-precision machining technology is promising for potential applications in the hydrophobic surface field [8]. Compared with other methods, high speed micro-milling process receives more attention due to its high machining accuracy, processing efficiency, simple preparation, relatively low cost and fewer limitations on processing materials [9].

In order to understand the nature of the hydrophobicity, the formation mechanism and the wetting phenomena, quantitative research has been carried out [10-11]. The Wenzel model [12] and the Cassie-Baxter model [13] have been used to interpret the phenomenon that the rough surface can increase the contact angle. However, the $\mathrm{CAH}$ in these two situations is far different with each other. Johnson and Dettre [14] found that when the surface roughness is relatively small, the droplet resting on the surface is in the Wenzel state. The CAH increases significantly with the increase of surface roughness. However, the $\mathrm{CAH}$ decreases rapidly when the surface roughness exceeds a certain value. The rapid decrease of the $\mathrm{CAH}$ is due to the fact that the droplet has changed into the Cassie-Baxter state. Compared with the Wenzel state, the trapped air 
in a rough surface can reduce the adhesion between the liquid droplet and solid surface for the Cassie-Baxter state. Suzuki et al. [15] evaluated the dynamic CAH of water droplets on a silicon surface that had been treated with fluoroalkyl silane. Results implied that the sliding acceleration of the water droplets on hydrophobic surfaces is controllable by changing the pattern structure of the surface and its chemical composition. Eral et al. [16] proposed a brief introduction of the $\mathrm{CAH}$ starting from a description of the physical phenomena. The model for implementing the CAH into relevant physical phenomena was then introduced. The influences of the $\mathrm{CAH}$ on several physical phenomena relevant for industrial applications such as sliding drops, coffee stain phenomenon, curtain and wire coating techniques were explained. Mundo and Palumbo [17] pointed out that the measurements of the $\mathrm{CAH}$ included static mode and dynamic mode. The static mode can be realized by simply placing a liquid drop on the surface. The dynamic mode was consistent in forcing the probe liquid to advance and retract, and then recording the angles of the liquid front corresponding to these variations.

Several researchers studied various theoretical bases for analytical models to investigate the effects of structure on surface hydrophobic properties. Miwa et al. [18] investigated the relationships between the sliding angle, the contact angle and the surface structure. A mathematical description of the relationships among them has been established. Results showed that the sliding angles of water droplets decrease with increasing contact angles. The surface structures, which can trap air, are important for the preparation of low sliding angle surfaces. Lv et al. [19] established an explicit analytical model to predict the sliding angles based on the observed mechanisms. With the proposed model, the sliding angle was determined by the fraction of water-solid interface area and Young's contact angle. A number of pillar and channel structured surfaces with different area ratios and different sizes have been investigated. Wang et al. [20] and Cai et al. [21] investigated the dynamic wetting behavior and water drops on micro-grooved surfaces. It was found that the dynamic advancing angle increases with the increase of the drop velocity and the receding angle decreases with it [20]. For droplets with different sizes, on the same substrate, 
the advancing angle and the receding angle slightly changed, but the $\mathrm{CAH}$ basically remained unchanged. Zhang et al. [22] proposed a three dimensional model based on the scanning electron microscopy of cicada wings to determine and explain the $\mathrm{CAH}$ in quantity. However, the established model was limited to explain some special circumstances which contain a number of empirical parameters.

This paper aims to investigate the effect of different micro-structures on hydrophobicity of surfaces based on the analysis of a number of hydrophobic surfaces fabricated under micro-milling process. The qualitative analysis based on experiments was conduct to reveal the impact mechanism of the structural parameters on hydrophobic performance. A comprehensive hydrophobic theory was established to explain the quantitative relationship between micro-structures and wetting performance of the rough surface. The established dynamic methodology model can clarify the importance of geometric scales on preparing a superhydrophobic surface. The established theoretical model can also be used to explain the wettability of rough surface and provide guidance on the preparation of superhydrophobic surfaces. Fig. 1 shows the general framework of this research.

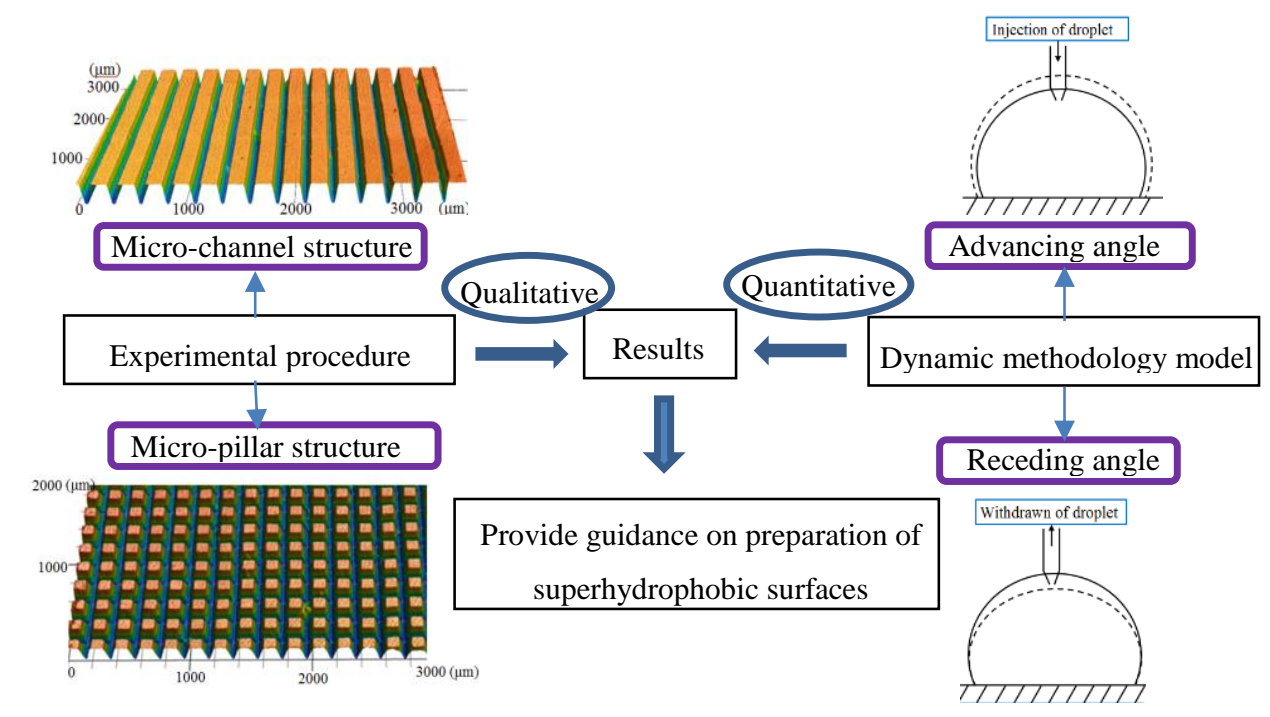

Fig. 1 Scope of this research

\section{Experiments}

\subsection{Micro-structure selection}


The advancing angle is the contact state when the droplet resting on a rough surface is about to move with the volume of droplet increasing as shown in Fig. 2. The receding angle is the contact state when the droplet resting on a rough surface is about to move with the volume of droplet decreasing. The advancing angle is always larger than the receding angle. $\mathrm{CAH}$ is the difference between the two angles. The magnitude of the $\mathrm{CAH}$ represents the degree of difficulty for a droplet to separate from the surface. As the $\mathrm{CAH}$ increases, it becomes more difficult for the droplet to separate from the surface. When the CAH is small to a certain level, it is easy for the droplet to separate from the surface, and in this case the surface has the properties of self-cleaning [23-24].
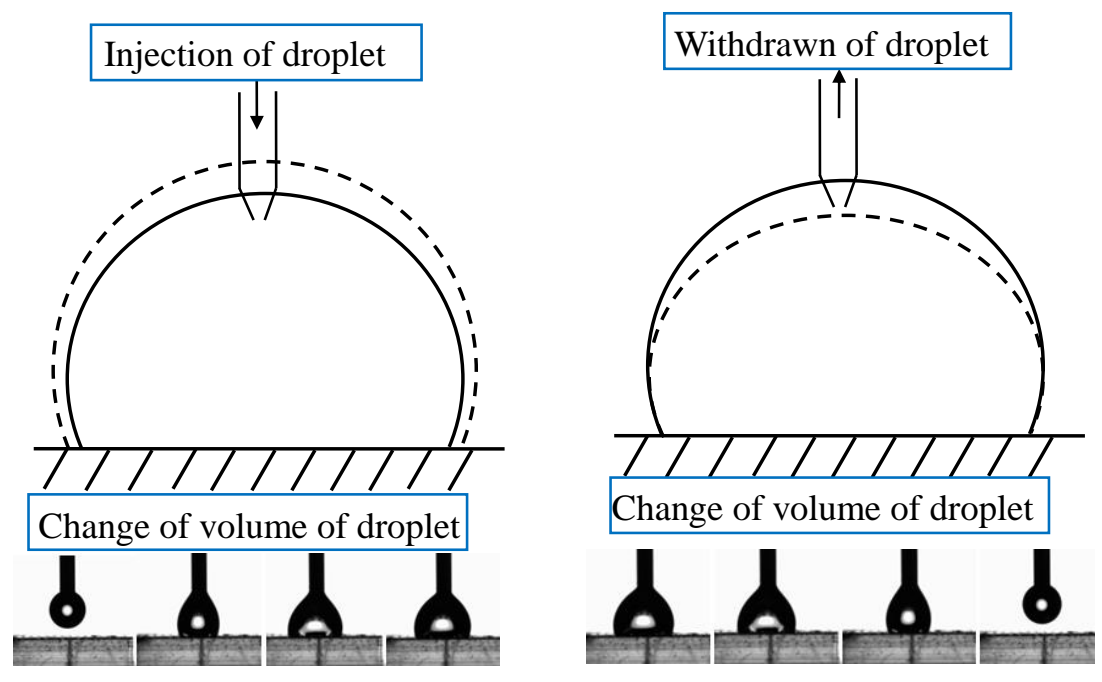

(a) Advancing state of droplet on rough surface (b) Receding state of droplet on rough surface

Fig. 2 Dynamical motion of droplet on rough surface

In this paper, two different micro-bump structures including micro-channel and micro-pillar structures are fabricated and analyzed. This paper proposes a 3-dimensional channel and a 3-dimensional pillar structure illustrated in Fig. 3 (a) and Fig. 3 (b), respectively. These structures can be simplified into a 2-dimensional system by analyzing the system along specific planes, e.g. at $y=0$, as illustrated in Fig. $3(\mathrm{c})$. 


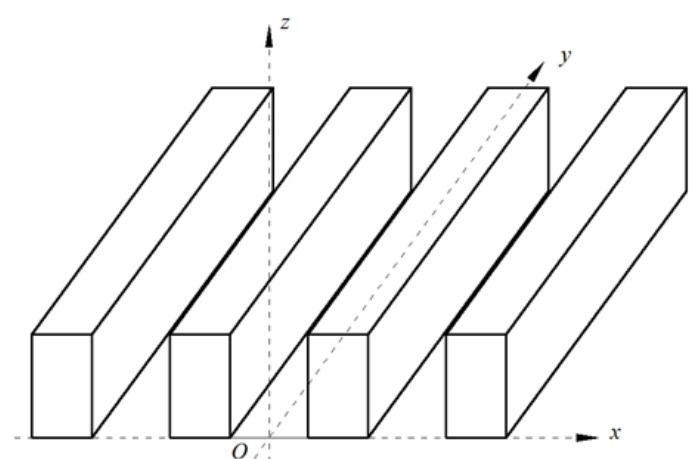

(a) 3-dimensional micro-channel structure

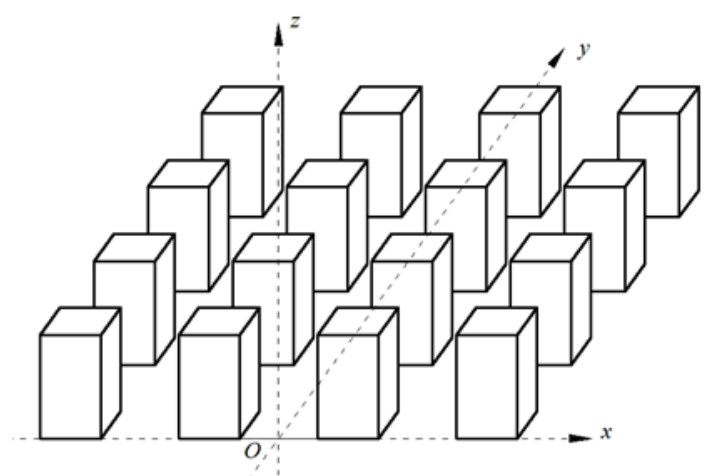

(b) 3-dimensional micro-pillar structure
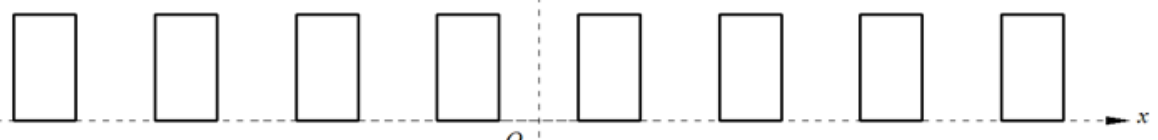

(c) Schematic cross section of micro-texture

Fig. 3 Typical channel and pillar surface micro-structure

\subsection{Experiments procedure}

The material removal process strongly influences the functionality of the machined surfaces. Micro-milling process is the physical treatment for the surface. The material removal can be realized based on the nature of phenomena of mechanical forces. Micro-milling process is believed to be able to get desirable shapes, geometrical accuracy and surface integrity to the maximum extent. Additionally, by using micro-milling process, there are lower environmental impacts than the surface modification approaches including materials treatments.

In this paper, experimental work has been carried out to investigate the effect of different micro-structures on advancing and receding angles through micro-milling process. PMMA was selected as workpiece to reduce the manufacturing error and defects for its characteristics of good transparency and easy to process. The workpiece was machined on a KERN 2522 micro-milling center as shown in Fig. 4 [25]. The cutting tool selected was a double-edged solid cemented carbide micro-milling cutter with diameter of $0.1 \mathrm{~mm}$. The experiments were carried out with a constant feed rate $(600 \mathrm{~mm} / \mathrm{min})$ and a constant spindle rotation speed (30000r/min). 


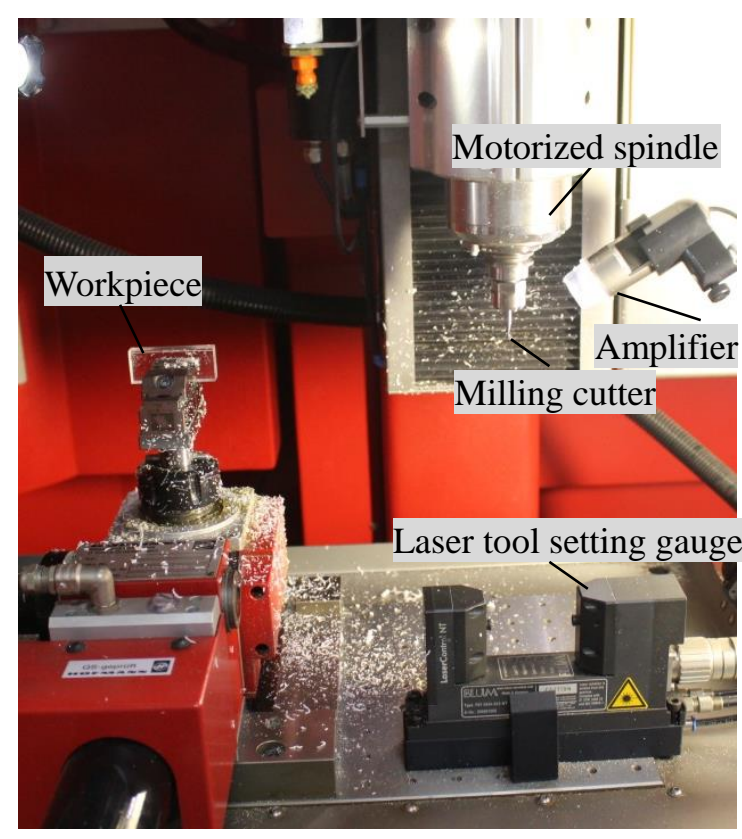

Fig. 4 Micro-milling center

The micro-channel structure and micro-pillar structure were machined as shown in Fig. 5. The micro-channel structure was completed through one pass in vertical direction and the micro-pillar structure was completed through two passes in vertical and horizontal directions.

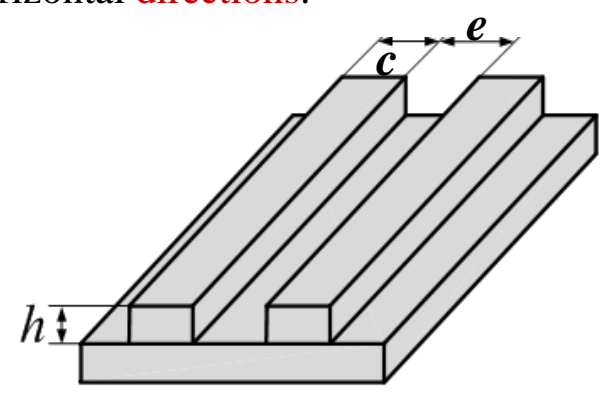

(a)micro-channel structure

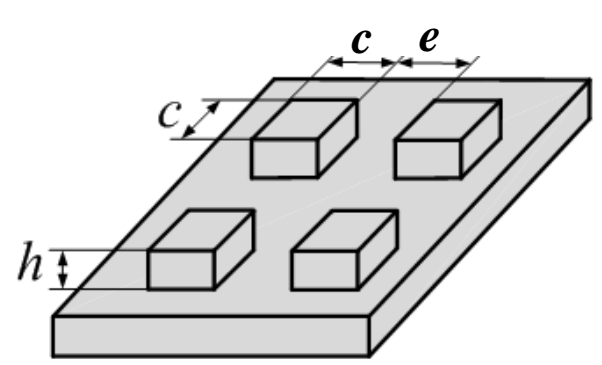

(b) micro-pillar structure

Fig. 5 Micro-channel structure and micro-pillar structure

When the machining process is completed, the finished surface has to be deburred to eliminate the effect of burrs on contact angle. At first, the machined surface was lightly brushed to remove most burrs with a $0.05 \mathrm{~mm}$ ultrafine brush. Then the machined surface was treated with alcohol ultrasonic cleaning for 30 minutes. Fig. 6 shows the comparison graph of the surfaces before and after burr removal. Finally, the machined surface was washed by de-ionized water in ultrasonic cleaning tank to remove impurities on the surface. 

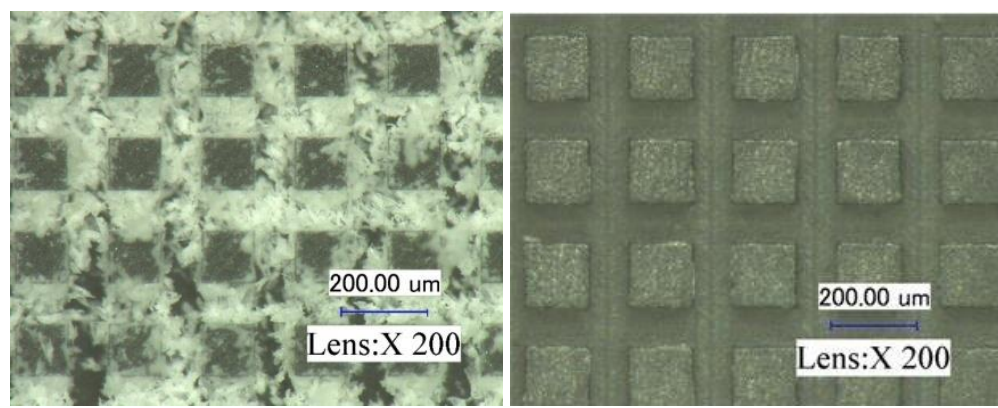

(a)No deburring surface (b) Deburring surface

Fig. 6 Comparison graph before and after burr removal

The static and dynamic contact angles were obtained through an optical contact angle measuring instrument. In the process of measurement, the workpiece was fixed on the measuring platform. The test liquid is deionized water with density $\rho=996 \mathrm{~kg} / \mathrm{m}^{3}$, surface tension $\sigma=0.07275 \mathrm{~N} / \mathrm{m}$, viscosity $\mu=0.001 \mathrm{~kg} / \mathrm{ms}$, and the initial droplet volume is $2 \mu \mathrm{L}$ [25]. Before the cutting experiments, the un-machined original sample was tested, and the average contact angle was about $80^{\circ}$.

Fig. 7 plots the measured advancing contact angle and receding contact angle as a function of a droplet volume on a micro-pillar structure with $c=150 \mu \mathrm{m}$, and $e=h=100 \mu \mathrm{m}$. The advancing and receding angle were measured through Pendant Drop method by the way of increasing or reducing the volume of the droplet. For the advancing angle, the droplet was gently deposited on the substrate and increased in steps through an automatic dispensing syringe. When a small drop is deposited on the surface, a static contact angle was formed between the advancing and receding values for the rough substrate. The advancing angle was measured after each 0.5 micro-Liter volume increment. The drop volume was increased up to about $5 \mu \mathrm{L}$. As the drop volume increased, the apparent angle increases until it reaches the maximum static angle which is defined as advancing angle. Once the advancing angle is reached, further increase in volume does not significantly change the apparent angle of the droplet. Fig. 7 gives an advancing angle $137^{\circ}$ for a droplet on the rough substrate. Receding contact angle measurements were then conducted by removing water from the droplet in steps. The apparent contact angle was measured after each volume reduced. For the receding angle, the volume reduction began from the last drop obtained in the advancing angle. The receding angle is found to be about $130^{\circ}$. The 
difference between the advancing and receding values is a measure of the hysteresis.

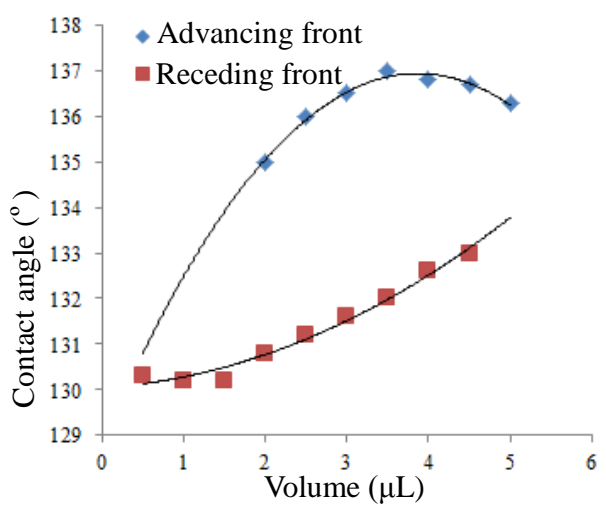

Fig. 7 Advancing and receding contact angle measurements for a droplet

\section{Experimental results analysis}

3.1 CAH analysis for micro-channel and micro-pillar structures

The samples of micro-channel structures were processed into 10 groups with $100 \mu \mathrm{m}$ constant height of channel and $100 \mu \mathrm{m}$ constant width of valley.

Table 1 Processing parameters for micro-structure

\begin{tabular}{lcccccccccc}
\hline Number of sample & 1 & 2 & 3 & 4 & 5 & 6 & 7 & 8 & 9 & 10 \\
\hline Width of peak/ $\mu \mathrm{m}$ & 25 & 50 & 75 & 100 & 125 & 150 & 175 & 200 & 225 & 250
\end{tabular}

The processing parameters were shown in Table 1. In order to investigate the effects of micro-structure on wetting behavior of hydrophobic surface, the width of peak increased arithmetically from $25 \mu \mathrm{m}$ to $250 \mu \mathrm{m}$.

Fig. 8 shows the obtained micro-channel structure under optical microscope.
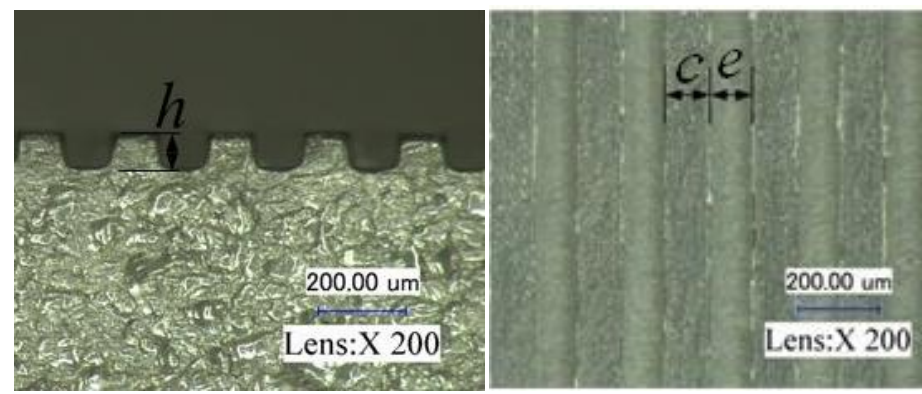


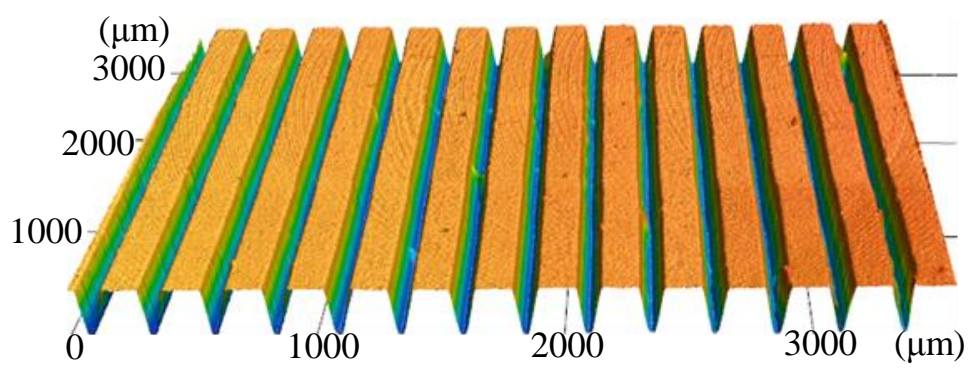

Fig. 8 Obtained micro-channel structure under optical microscope

The micro-channel structures can create droplet anisotropy [26]. Hence, the dynamic contact angles in micro-channel directions and its perpendicular directions are different. In this paper, only the dynamic contact angle in the direction of micro-channel was investigated which got the same projection as micro-pillar structure in $x \mathrm{O} z$ plane as shown in Fig. 3.

The samples of micro-pillar structure processed of a total of 10 groups with a constant height and spacing of pillar of $100 \mu \mathrm{m}$. The width of pillar increases from $25 \mu \mathrm{m}$ to $250 \mu \mathrm{m}$ arithmetically. The micro-pillar structures are isotropic. Hence, the dynamic contact angles are identical in two directions.

Fig. 9 shows the obtained micro-pillar structure under optical microscope.
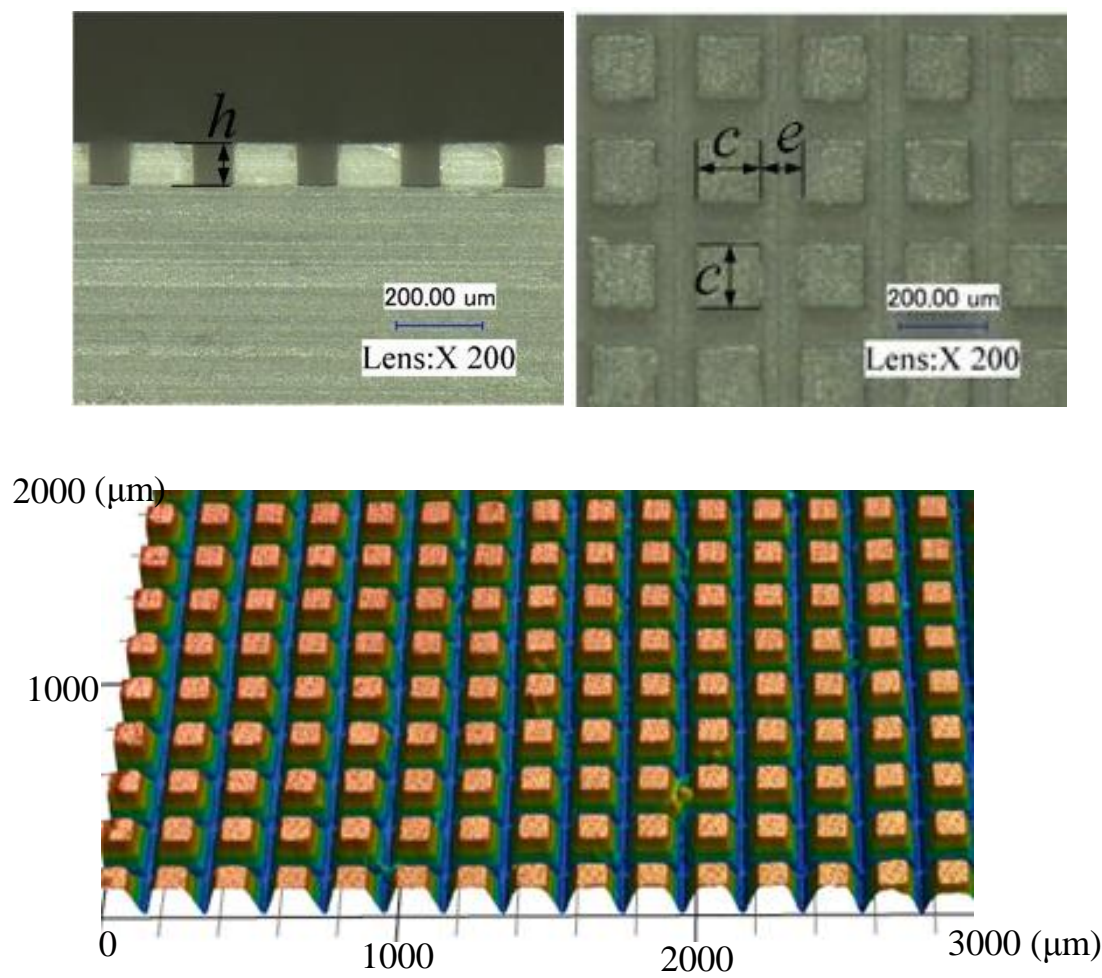

Fig. 9 Obtained micro-pillar structure under optical microscope 
Fig. 10 shows the comparison of the dynamic contact angles for two different micro-structures. Values of contact angles were obtained by averaging over 6 measurements and error bars were added in Fig. 10.

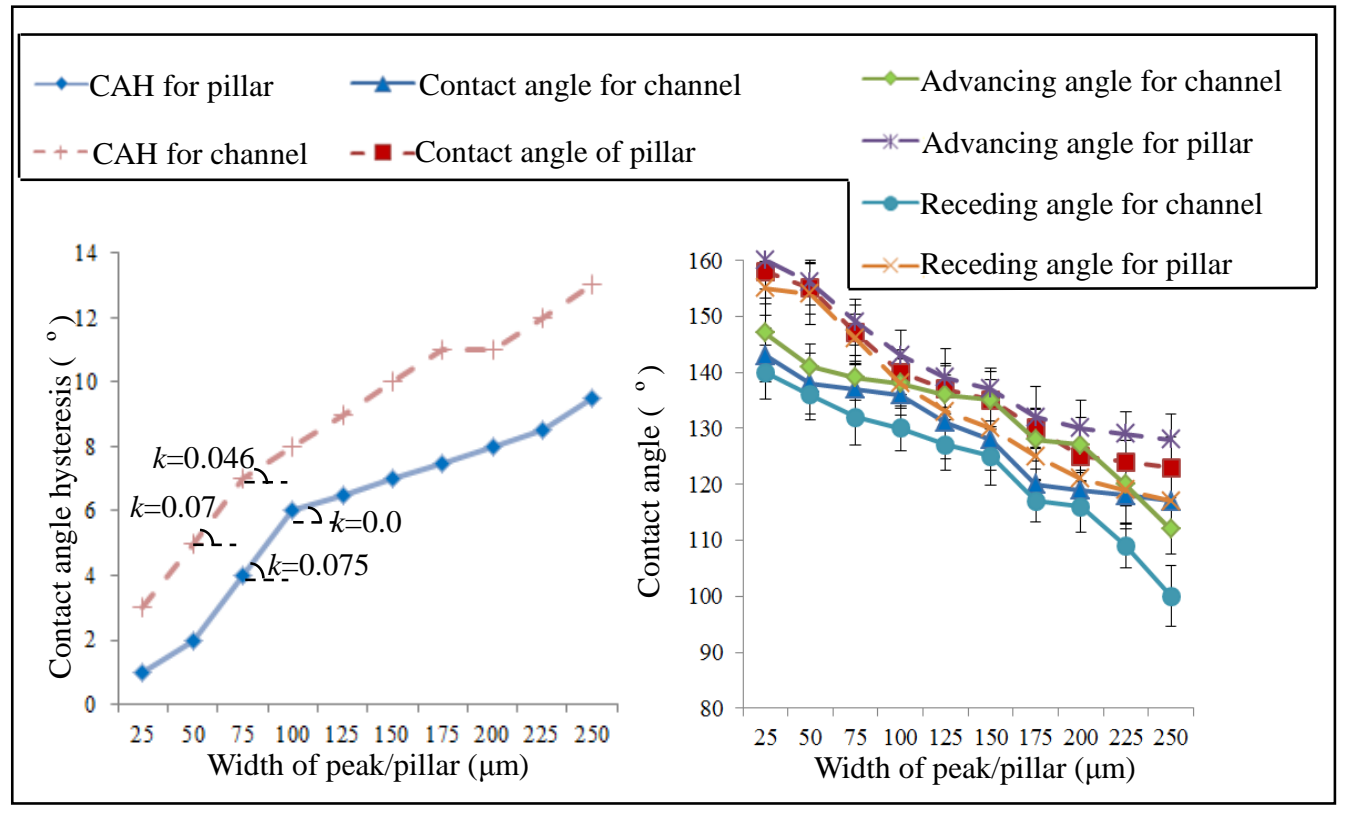

(a) $\mathrm{CAH}$ changes with width of peak/pillar (b) Contact angle changes with widths

Fig. 10 Effect of micro-structure on dynamical contact angle. Error bars indicate SD ( $n=6$,

$$
\mathrm{p}<0.05)
$$

By comparing the experimental results as shown in Fig. 10, it can be seen that the advancing and receding angles decrease with, while the $\mathrm{CAH}$ increases with, the increase of the peak width for both micro-channel and micro-pillar structures.

As Fig.10 shows, when width of peak is smaller than $75 \mu \mathrm{m}$, the contact angle is very large and the $\mathrm{CAH}$ becomes very small for micro-channel structure. It can be explained by the fact that with the increase of width of peak, solid fraction increases, which in turn increases the $\mathrm{CAH}$ and weakens the hydrophobic of the surface. Based on analysis of the curve's slope, as Fig. 10(a) shows, the same conclusions can be drawn. When the width is no more than $75 \mu \mathrm{m}$, the slope $k$ is about 0.07 . With increase of the width, $k$ decreases to 0.046 . It means the variation of the contact angle and the $\mathrm{CAH}$ are not prominent as the peak width increases.

For the pillar structure, as shown in Fig. 10, the critical value for the width of pillar is $100 \mu \mathrm{m}$. When it is larger than $100 \mu \mathrm{m}$, the contact angle and the CAH change 
slowly. When it is smaller than $100 \mu \mathrm{m}$, the contact angle becomes very large and the $\mathrm{CAH}$ becomes very small, and the surface has changed to be a hydrophobic surface. This also can be explained in the view point of curve's slope, as shown in Fig. 10. When pillar width is no smaller than $100 \mu \mathrm{m}$, the slope $k$ is about 0.075 . With increase of width, $k$ decreases to 0.04 .

From Fig. 10, it can also be seen that for the same PMMA matrix, when micro parameters keep consistent, the pillar structure has larger contact angle and smaller CAH than that of channel structure which can make the surface more hydrophobic. Qualitative analysis was conducted based on the droplet baseline on micro-structures. 3.2 Qualitative analysis of droplet baseline

The contact line means one or more space curves for solid, liquid and vapor phase contact with each other [27]. The motions of the contact lines for pillar and channel structure were investigated as shown in Fig. 11.

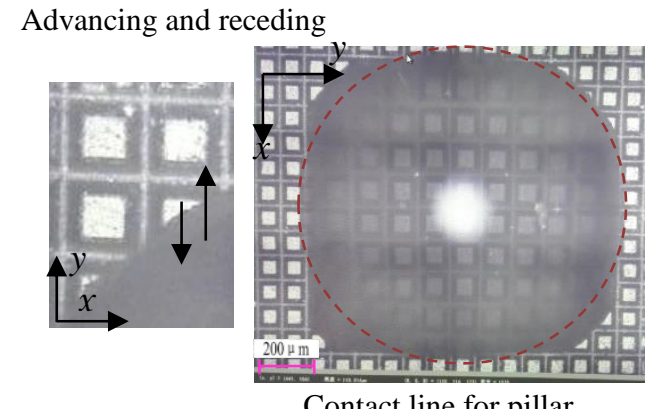

Contact line for pillar

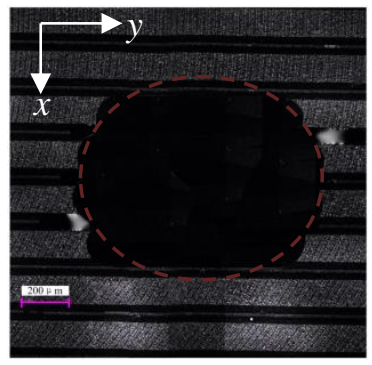

Contact line for channel
Advancing and receding

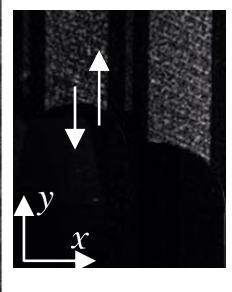

Fig. 11 Bottom view of droplet baseline for pillar and channel surfaces

In Fig.11, it can be seen that for the droplet resting on pillar substrate, it is almost a sphere, and the contact area for the droplet and the surface is reduced. The interaction between solid and liquid is also decreased. While for channel surface, the shape of the contact line slightly deviates from circular. For a droplet resting on pillar surfaces, the movement is independent with sphere, and its main motion is characterized by rolling style instead of sliding. That is the reason why the droplet has larger contact angle and smaller $\mathrm{CAH}$ on pillar surface. According to the principle of optimization, for hydrophobic surface with the maximum apparent contact angle and the minimum $\mathrm{CAH}$, pillar structure is more favorable for hydrophobicity.

Fig. 12 shows a sequence of snapshots of the contact line near the edge of the 
droplet.

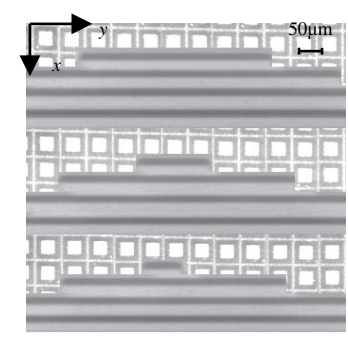

Fig. 12 Contact line near the edge of the droplet for pillar structure

As Fig. 12 shows, the line recedes in abrupt steps of the size of $c+e$ in $x$ direction, and remains pinned for a period before next step. While in this situation, the contact line slides in $y$ direction, and the contact line is intermittent. According to Reference [28], when the contact line is continuous, the droplet on solid surface is not easy to roll, and the $\mathrm{CAH}$ is larger; when the contact line is intermittent, the droplet on solid surface is easy to roll, and the $\mathrm{CAH}$ is smaller. In most situations, this is wrought by the existence of composite wetting mechanism.

\section{Dynamic modeling of contact angle hysteresis}

To quantitative analysis the effect of micro-structure and parameters on the $\mathrm{CAH}$, five key states of the droplet including the initial, pre-forward, forward, pre-backward and backward were taken as the objects to investigate the wetting phenomena of hydrophobic surfaces. During the research, the three-dimensional water drop was analyzed in a two-dimensional manner through cross section views of the droplet. The cross section for the water droplet resting on micro-structures is shown in Figure 13.

\subsection{Advancing state}

Fig. 13 shows the droplet in transition to advancing state. When water is injected and the droplet comes forward. In Fig. 13, $h$ is the height of the channel, $c$ represents the width of peak and $e$ represents the valley width. 


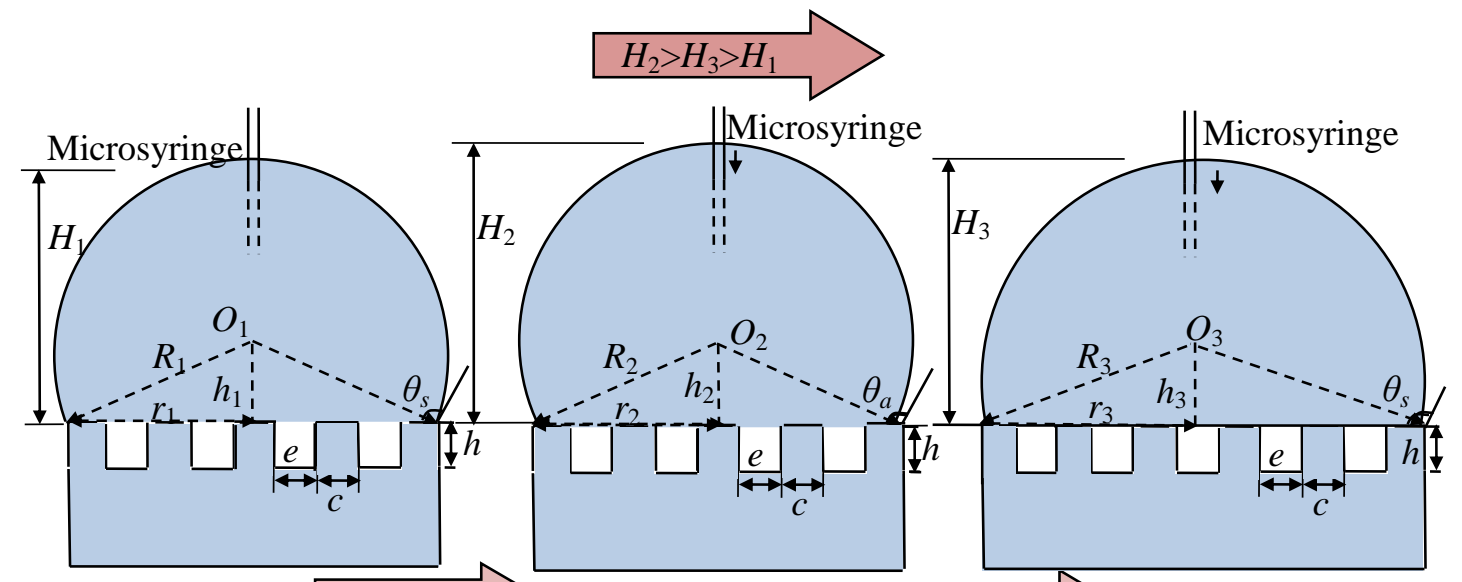

(a) Initial state $\quad r_{1}=r_{2}, \mathrm{v}_{1}<\mathrm{v}_{2}>$ (b) Pre-advancing state $r_{3}>r_{2}, \mathrm{v}_{2}=\mathrm{v}_{3}>$ (c) Advancing state

Fig.13 Advancing state for a droplet

Fig. 13(a) shows the initial stable state with apparent contact angle $\theta_{s} . H_{1}$ is the height of droplet at initial state. $O_{1}$ is the center of droplet. $r_{1}$ is the radius of contact line among liquid, vapor and solid, and $h_{1}$ is the distance between droplet center to solid surface. $R_{1}$ is the radius of the initial droplet, which can be calculated through sphere volume formula: $R_{1}=\sqrt[3]{\frac{3 V}{4 \pi}}$, where $V$ is the volume of the initial droplet.

According to Fig. 13(a), there is:

$$
r_{1}=R_{1} \sin \left(\pi-\theta_{s}\right)=R_{1} \sin \theta_{s}
$$

With water added, Fig. 13(b) shows pre-advancing state with an advancing angle $\theta_{a}$. In Fig. 13(b), $\mathrm{H}_{2}$ is the height of droplet at pre-advancing state. $\mathrm{O}_{2}$ is the center of droplet. $r_{2}$ is the radius of contact line among liquid, vapor and solid, and $h_{2}$ is the distance between the droplet center and the solid surface for pre-advancing state.

In this situation, the volume of the droplet keeps increasing but the position of the drop does not change. Hence, $r_{1}$ equals to $r_{2}$.

According to Fig. 13 (b), there is:

$$
r_{2}=r_{1}=R_{1} \sin \theta_{s}
$$

The volume of droplet can be calculated as:

$$
V_{2}=\frac{4}{3} \pi R_{2}{ }^{3}-\pi\left(2 R_{2}-H_{2}\right)^{2}\left(R_{2}-\frac{2 R_{2}-H_{2}}{3}\right)
$$




$$
\begin{gathered}
H_{2}=R_{2}+h_{2} \\
R_{2}=\frac{r_{2}}{\sin \theta_{a}}=\frac{r_{1}}{\sin \theta_{a}} \\
h_{2}=\sqrt{R_{2}^{2}-r_{2}^{2}}=\sqrt{\left(\frac{r_{1}}{\sin \theta_{a}}\right)^{2}-r_{1}^{2}}=r_{1} \cot \theta_{a} \\
V_{2}=\frac{4}{3} \pi\left(\frac{r_{1}}{\sin \theta_{a}}\right)^{3}-\pi\left(\frac{r_{1}}{\sin \theta_{a}}-r_{1} \cot \theta_{a}\right)^{2}\left(\frac{r_{1}}{\sin \theta_{a}}-\frac{1}{3}\left(\frac{r_{1}}{\sin \theta_{a}}-r_{1} \cot \theta_{a}\right)\right)=\frac{1}{3} \pi\left(\frac{R_{1} \sin \theta_{s}}{\sin \theta_{a}}\right)^{3}\left(2+3 \cos \theta_{a}-\cos ^{3} \theta_{a}\right)
\end{gathered}
$$

Fig. 13 (c) shows the advancing state while the droplet already moved, and the contact angle backed to the apparent contact angle $\theta_{s}$. In Fig. 13(c), $H_{3}$ is the height of droplet at the advancing state. $O_{3}$ is the center of droplet. $r_{3}$ is the radius of contact line among liquid, vapor and solid, and $h_{3}$ is the distance between the droplet center and the solid surface for the advancing state.

In this situation, water cannot be added anymore, so the volume of the droplet is equal to that of situation of pre-advancing state.

And in Fig. 13 (c), there is: $r_{3}=R_{3} \sin \theta_{s}$

The volume of droplet can be calculated as:

$$
V_{3}=\frac{1}{3} \pi\left(\frac{r_{3}}{\sin \theta_{s}}\right)^{3}\left(2+3 \cos \theta_{s}-\cos ^{3} \theta_{s}\right)
$$

For $V_{3}=V_{2}$,

$$
\frac{1}{3} \pi\left(\frac{r_{3}}{\sin \theta_{s}}\right)^{3}\left(2+3 \cos \theta_{s}-\cos ^{3} \theta_{s}\right)=\frac{1}{3} \pi\left(\frac{R_{1} \sin \theta_{s}}{\sin \theta_{a}}\right)^{3}\left(2+3 \cos \theta_{a}-\cos ^{3} \theta_{a}\right)
$$

The relationship between $R_{3}$ and $R_{1}$ can be expressed as:

$$
r_{3}=r_{1} \frac{\sin \theta_{s}}{\sin \theta_{a}} \sqrt[3]{\frac{2+3 \cos \theta_{a}-\cos ^{3} \theta_{a}}{2+3 \cos \theta_{s}-\cos ^{3} \theta_{s}}}
$$

In Eq. $10, \cos ^{3} \theta_{a}$ and $\cos ^{3} \theta_{s}$ have little effect on results, hence, it can be expressed as:

$$
r_{3}=r_{1} \frac{\sin \theta_{s}}{\sin \theta_{a}} \sqrt[3]{\frac{2+3 \cos \theta_{a}}{2+3 \cos \theta_{s}}}
$$


According to Fig. 14, the parameters of droplet rest on micro-structured surface can also be got as following:

$$
\begin{gathered}
r_{1}=\frac{n e+(n+1) c}{2} \\
r_{3}=\frac{(n+1) e+(n+2) c}{2}
\end{gathered}
$$

where $n$ represents the number of valley.

$$
r_{3}-r_{1}=\frac{e+c}{2}
$$

Substituting Eq. 11 into Eq. 14,

$$
r_{1} \frac{\sin \theta_{s}}{\sin \theta_{a}} \sqrt[3]{\frac{2+3 \cos \theta_{a}}{2+3 \cos \theta_{s}}}-r_{1}=\frac{e+c}{2}
$$

The relationship between advancing angle $\theta_{a}$ and apparent contact angle $\theta_{s}$ can be expressed as:

$$
\begin{aligned}
& \frac{\sin \theta_{s}}{\sin \theta_{a}} \sqrt[3]{\frac{2+3 \cos \theta_{a}}{2+3 \cos \theta_{s}}}=\frac{e+c}{2 r_{1}}+1 \\
& \frac{\sin \theta_{s}}{\sin \theta_{a}} \sqrt[3]{\frac{2+3 \cos \theta_{a}}{2+3 \cos \theta_{s}}}=\frac{e+c}{2 R_{1} \sin \theta_{s}}+1
\end{aligned}
$$

When apparent contact angle $\theta_{s}$ is known, Eq. 17 can be used to calculate the advancing angle.

\subsection{Receding state}

Fig.14 shows the receding state. At the receding state, water is being extracted and the droplet is receding. 


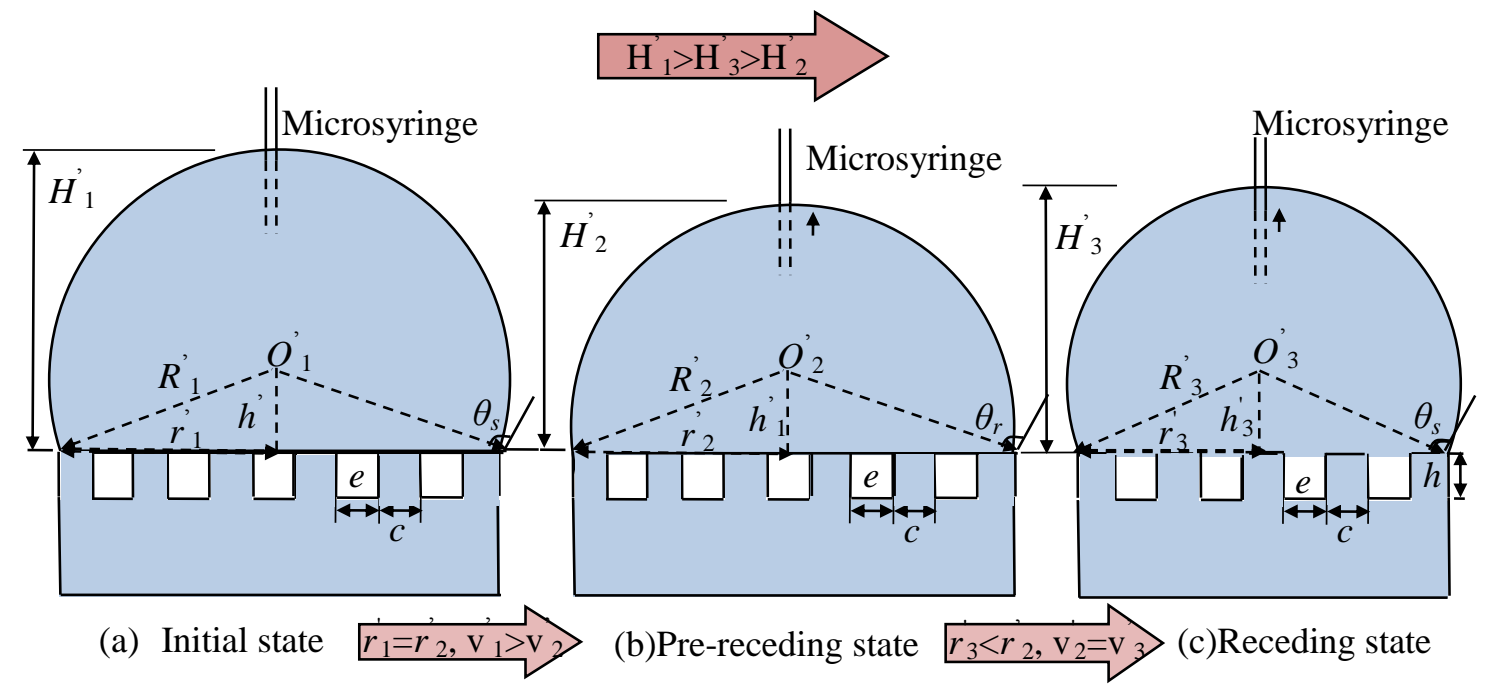

Fig. 14 Receding state for droplet

Fig. 14 (a) shows the initial stable state with apparent contact angle $\theta_{s}$.

While in Fig. 14 (a), there is:

$$
r_{2}^{\prime}=r_{1}^{\prime}=R_{1}^{\prime} \sin \left(\pi-\theta_{s}\right)=R_{1} \sin \theta_{s} \text { for }\left(R_{1}^{\prime}=R_{1}, r_{1}^{\prime}=r_{1}\right)
$$

In Fig. 14 (c), the receding radius can be expressed as:

$$
r_{3}^{\prime}=r_{1} \frac{\sin \theta_{s}}{\sin \theta_{r}} \sqrt[3]{\frac{2+3 \cos \theta_{r}}{2+3 \cos \theta_{s}}}
$$

The parameters of droplet resting on micro-structured surface can be got as following:

$$
\begin{aligned}
& r_{1}=\frac{n e+(n+1) c}{2} \\
& r_{3}^{\prime}=\frac{(n-1) e+n c}{2} \\
& r_{1}-r_{1} \frac{\sin \theta_{s}}{\sin \theta_{r}} \sqrt[3]{\frac{2+3 \cos \theta_{r}}{2+3 \cos \theta_{s}}}=\frac{e+c}{2} \\
& \frac{\sin \theta_{s}}{\sin \theta_{r}} \sqrt[3]{\frac{2+3 \cos \theta_{r}}{2+3 \cos \theta_{s}}}=1-\frac{e+c}{2 r_{1}}
\end{aligned}
$$

When apparent contact angle $\theta_{s}$ is known, Eq. 23 can be used to calculate the receding angle.

In general situation, assuming the entire composition and micro-nano structure keep consistent, the relationship between the contact angles on hydrophobic surface 
can be expressed as:

$$
\theta_{a}>\theta_{s}>\theta_{r}
$$

Normally the apparent contact angle is no more than $170^{\circ}$. Stepping technique was adopted to derivate the expression for the apparent contact angle, the advancing angle and the receding angle in this range. The numerical solution for advancing angle and receding angle as shown in Eq.17 and Eq.23 can be expressed as following:

$$
\begin{aligned}
& \sin \theta_{a}=\left(0.95-\sqrt[3]{\frac{e+c}{2 R_{1}}}\right) \sin \theta_{s} \\
& \sin \theta_{d}=\left(1.02+\sqrt[3]{\frac{e+c}{2 R_{1}}}\right) \sin \theta_{s}
\end{aligned}
$$

The error for the predicted advancing and receding angles caused by constant can be ignored due to the relationship between apparent contact angles, which can be obtained through arcsine operations. For $\theta_{\alpha}$ and $\theta_{s}$ were in the second quadrant, they can calculated through Eq. 26 and Eq. 27.

$$
\begin{aligned}
& \theta_{a}=\pi-\arcsin \left[\left(0.95-\sqrt[3]{\frac{e+c}{2 R_{1}}}\right) \sin \theta_{s}\right] \\
& \theta_{d}=\pi-\arcsin \left[\left(1.02+\sqrt[3]{\frac{e+c}{2 R_{1}}}\right) \sin \theta_{s}\right]
\end{aligned}
$$

Hence, the CAH can be expressed as:

$C A H=\theta_{a}-\theta_{d}=\pi-\arcsin \left[\left(0.95-\sqrt[3]{\frac{e+c}{2 R_{1}}}\right) \sin \theta_{s}\right]-\left\{\pi-\arcsin \left[\left(1.02+\sqrt[3]{\frac{e+c}{2 R_{1}}}\right) \sin \theta_{s}\right]\right\}$

According to Cassie-Baxter model, for micro-channel structure, the actual ratio of solid-liquid contact area and the projection for the droplet projected on bottom can be expressed as:

$$
f_{b}=\frac{c}{c+e}
$$

Put $f_{b}$ into Cassie-Baxter model, the apparent contact angle for micro-channel structure can be calculated: 


$$
\cos \theta_{s}=-1+(1+\cos \theta) \times \frac{c}{e+c}
$$

For micro-pillar structure, the fraction of the solid/liquid interface $f_{\mathrm{b}}$ can be calculated through Eq. 37 according to Fig. 15.

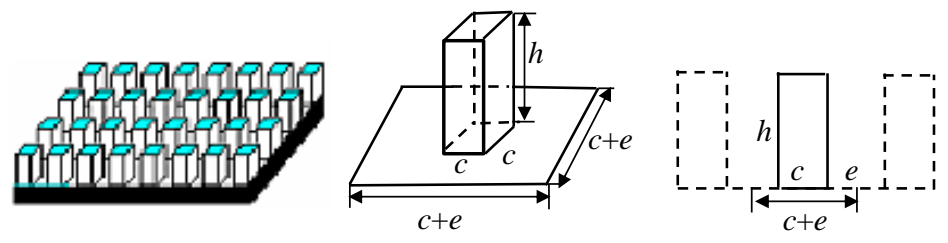

Fig. 15 Solid fraction for micro-pillar structure

$$
f_{b}=\frac{c^{2}}{(c+e)^{2}}
$$

Put $f_{b}$ into Cassie-Baxter model, the apparent contact angle for micro-pillar structure can be got:

$$
\cos \theta_{s}=-1+(1+\cos \theta) \times \frac{\mathrm{c}^{2}}{(\mathrm{c}+e)^{2}}
$$

\subsection{Prediction results analysis}

Substituting Eq. 30 and E. 32 into Eq. 26, Eq. 27 and Eq. 28, the graph demonstrating the relationship between advancing, receding angle, the $\mathrm{CAH}$ and area fraction for micro-channel and micro-pillar structures can be derived.

The effect of micro-structure parameters on the area fraction is shown in Fig. 16, where the area fraction is plotted against the width of peak according to Eq. 29 and Eq. 31 for micro-channel and micro-pillar structures respectively.

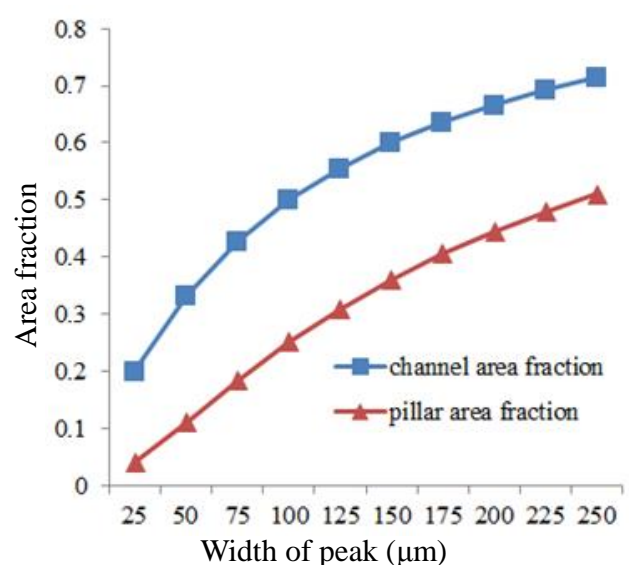

Fig. 16 Relationship between width of peak and area fraction

Fig. 17 shows the calculated contact angle and $\mathrm{CAH}$ according to the established 
theoretical model. The CAH shown in Fig. 17 (a) calculated with Eq. 28 was the difference between Eq.26 and Eq.27 (the advancing angle and the receding angle). In order to present it clearly, the difference was marked in Figure 17 (b) to correspond the value of the CAH in Fig. 17 (a).

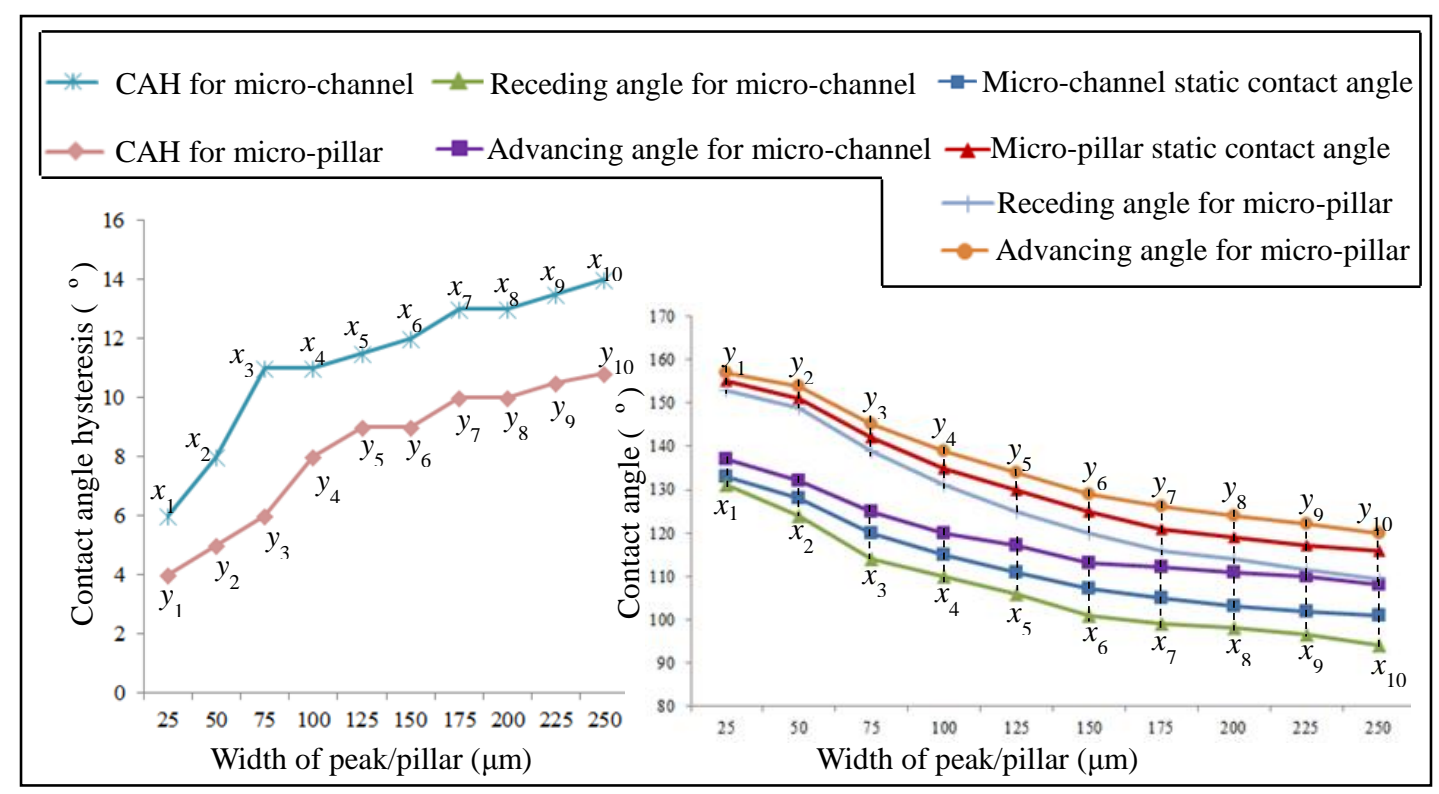

(a) CAH changes with width of peak/pillar (b) Contact angle changes with widths

Fig. 17 Relationship between width of peak/pillar and contact angle

In Fig. 17 (a), $x_{n}(\mathrm{n}=1 \sim 10)$ and $y_{n}(\mathrm{n}=1 \sim 10)$ represent the value of the calculated $\mathrm{CAH}$ for micro-channel and micro-pillar structures, which corresponds to the $x_{n}(\mathrm{n}=1 \sim 10)$ and $y_{n}(\mathrm{n}=1 \sim 10)$ in Fig. 17 (b) that is the difference between the calculated advancing angle and receding angle.

From Fig. 16 and Fig. 17, it can be seen that the area fraction increases with the width of micro-structure when volume of droplet remains constant. For micro-channel and micro-pillar structures, the apparent contact angles decrease with the increase of area fraction. When area fraction tends to 0 , the apparent contact angle is close to 180 o theoretically. From Fig. 17 (a), it can be seen that the CAH increases with the increase of area fraction. Meantime, the $\mathrm{CAH}$ and the area fraction are constraints with each other and show a similar trend, which means both of them have an increment or decrement operations at the same time.

From Fig. 16 and Fig. 17, it can be seen that the solid area fraction for micro-channel structure corresponds to 0.42 when width of peak is $75 \mu \mathrm{m}$. The 
apparent contact angle and the $\mathrm{CAH}$ is close to $130^{\circ}$ and $10^{\circ}$ respectively. As shown in Fig. 17 (b), when the width of peak is greater than $75 \mu \mathrm{m}$, the apparent contact angle and the $\mathrm{CAH}$ change slowly. Hence, solid fraction with the value of 0.42 will be the best choice for micro-channel structure. When the solid fraction is too low, the mechanical properties of the surface are difficult to achieve. With the area fraction increases, it is difficult to satisfy the requirements of hydrophobicity. For micro-pillar structure, when the width of peak is $100 \mu \mathrm{m}$, the solid area fraction corresponds to 0.25 , which is the critical value for machining of hydrophobic surface. These results confirmed the possibility of the transition from hydrophilic to hydrophobic.

To make intuitive comparisons between the experimental results and the calculated results, the results in Fig. 11 and Fig. 17 were incorporated into Fig. 18 as shown in following.

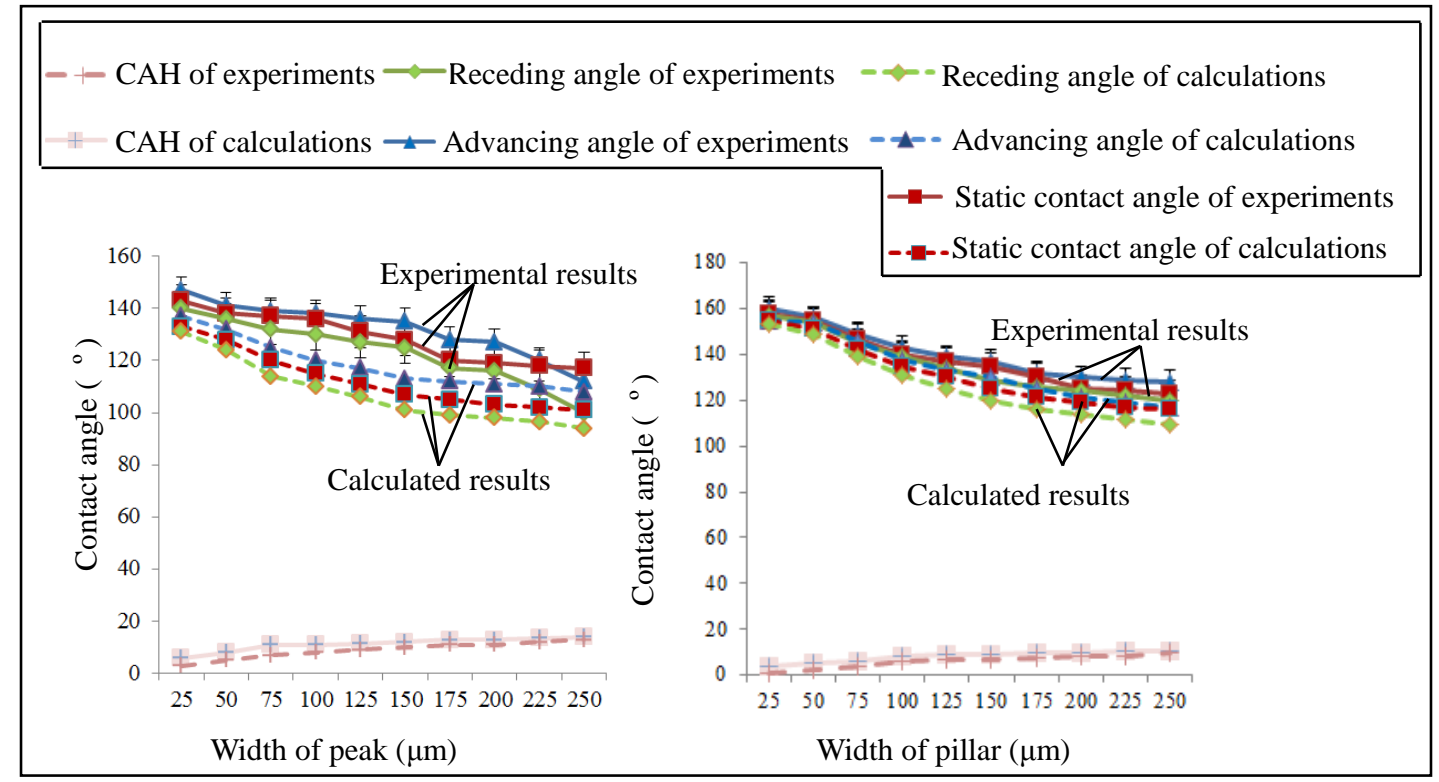

(a) Contace angle changes for channel structure (b) Contact angle changes for pillar structure Fig. 18 Comparison of experimental results and calculated results for micro-channel and micro-pillar structure

In Fig. 18, it can be seen that experiment results and prediction model results got good consistent with each other. The difference between experimental and prediction results mainly comes from two aspects. First, the vibration of the machine tool could create a difference between the design size and final size created features which can change the contact angle. The second is that, the advancing angle and receding angle were obtained by adding or reducing volume of the drop. In practice, it is difficult to 
insert the needle into the center of the droplet, which is another error source for the experimental results.

Based on the analysis, it can be concluded that the variety of area fraction can emphasize the effect of micro-structure on hydrophobic properties. It is an important aspect to select an appropriate micro-structure to improve the hydrophobic properties. It should also be noted that, the rough surface structure can endow hydrophobic properties for surface, but appreciatively leads to poor mechanical properties for the surface. Hence, there exist the optimal structure parameters for fabricating hydrophobic surfaces.

The trends of Fig. 18 agree with the results from Rodríguezvalverde [28], Lv [19], Xiu [29] and Wang [30] who also investigate the CAH on hydrophobic surfaces. The difference is that these established models contains a number of empirical parameters, while the prediction model established in this paper utilize numerical analysis and can be used to explain the wettability of rough surfaces.

\section{Conclusions}

In this paper, two different micro-structured hydrophobic surfaces were machined though micro-machining process. The advancing and receding angles were measured through Pendant Drop method by the way of increasing or reducing the volume of the droplet. A transient state equation has been established to describe the advancing and the receding motion of droplets resting on hydrophobic surfaces with the hysteresis phenomenon. The five key states of the droplet including the initial, pre-forward, forward, pre-backward, backward were taken as the objects of this research. The effects of structure parameters on hydrophobicity were discussed qualitatively and quantitatively. The results indicated that the hydrophobic properties of the surface depend on the micro-structure. The solid fraction played a decisive role in the transition from being hydrophilic to hydrophobic, which existed different degrees of difference for different morphologies. Smaller solid fraction resulted in a larger contact angle and a smaller CAH. For different micro-structures, the hydrophobicity of the surface could be controlled by adjusting the structure parameters. 


\section{Acknowledgement}

The authors would like to thank Professor Philip Mathew, Guest Professor, School of Mechanical Engineering, Shandong University for the help with the writing and improving the clarity of the paper.

The authors would like to acknowledge the financial support from the National Natural Science Foundation of China (51505255 and 51425503). The work was supported by grants from Tai Shan Scholar Foundation (TS20130922) and the Fundamental Research Funds of Shandong University.

\section{Reference}

[1] X.L. Tian, T. Verho, R.H.A. Ras, Moving superhydrophobic surfaces toward real-world applications, Sci. 352 (2016) 142-143.

[2] P.F. Hao, Z.H. Yao, X.W. Zhang, Study of dynamic hydrophobicity of micro-structured hydrophobic surfaces and lotus leaves, Sci. China Phys. Mech. 54 (2011) 675-682.

[3] A.L. Dubov, A. Mourran, M. Möller, O.I. Vinogradova, Contact angle hysteresis on superhydrophobic stripes, J. Chem. Phys. 141(2014)074710 1-6.

[4] N. Linder, A. Criscione, I.V. Roisman, H. Marschall, C. Tropea, 3D computation of an incipient motion of a sessile drop on a rigid surface with contact angle hysteresis, Theor. Comput. Fluid Dyn. 29 (2015)373-390.

[5] H.Y. Zhang, W. Li, G. Fang, A new model for thermodynamic analysis on wetting behavior of superhydrophobic surfaces, Appl. Surf. Sci. 258 (2012) 2707-2716.

[6] D.C.K. Chung, S.H. Huynh, S. Wang, X. Jiang, O.W. Liew, M. Muradoglu, T.W. Ng, A superhydrophobic manhole for drops, J. Mater. Chem. A. 5(2017)914-918.

[7] T. Vuong, B.H. Cheong, S.H. Huynh, M. Muradoglu, O.W. Liew, T.W. Ng, Drop transfer between superhydrophobic wells using air logic control, Lab Chip 15(2015)991-995.

[8] Y. Liang, L. Shu, W. Nastu, F. He, Anisotropic wetting characteristics versus roughness on machined surfaces of hydrophilic and hydrophobic materials, Appl. Surf. Sci. 331(2015)41-49.

[9] E. Wielewski, M.R. Arthington, C.R. Siviour, N. Petrinic, Characterising the effects of strain rate, crystallographic texture and direction of loading on the mechanical behavior of Ti-6Al-4V, J. Dynamic Behavior Mater. 1(2015)462-471.

[10] B. He, J. Lee, N.A. Patankar, Contact angle hysteresis on rough hydrophobic surfaces, Colloid. Surf. A. 248 (2004) 101-104. 
[11] W. Li, A. Amirfazli, A thermodynamic approach for determining the contact angle hysteresis for superhydrophobic surfaces, J. Colloid. Interf. Sci. 292 (2005) 195-201.

[12] R.N.Wenzel, Resistance of solid surfaces to wetting by water, Ind. Eng. Chem. Res. 28(8) (1936) 988-994.

[13] A.B.D. Cassie, S. Baxter, Wettability of porous surfaces, Trans. Faraday. Soc. 40(1944) 546-551.

[14] R.E. Johnson, R.H. Dettre, Contact angle, wettability and adhesion, Washington DC, 1964.

[15] S. Suzuki, A. Nakajima, Y. Kameshima, Elongation and contraction of water droplet during sliding on the silicon surface treated by fluoroalkylsilane, Surf. Sci. 557 (2004) 163-168.

[16] H.B. Eral, D.J.C.M.'t Mannetje, J.M. Oh, Contact Angle Hysteresis: a review of fundamentals and applications, Colloid. Polym. Sci. 291 (2013) 247-260.

[17] R.Di. Mundo, F. Palumbo, Comments regarding "an essay on contact angle measurements", Plasma. Process. Polym. 8 (2011) 14-18.

[18] M. Miwa, A. Nacajima, A. Fujishima, K. Hashimoto, T. Watanabe, Effects of the surface roughness on sliding angles of water droplets on superhydrophobic surfaces, Langmuir. 16 (2000) 5754-5760.

[19] C.J. Lv, C.W. Yang, P.F. Hao, Sliding of water droplets on microstructured hydrophobic surfaces, Langmuir. 26.11 (2010) 8704-8708.

[20] X. Wang, M. Ashiqur Rahman, A.M. Jacobi, P.S. Hrnjak, Dynamic wetting behavior and water drops on microgrooved surfaces, Heat Trans. Eng. 34(13)(2013)1088-1098.

[21] T. Cai, Z. Jia, J. He, W. Lei, Hysteresis characteristics of droplets on inclined microstructured hydrophobic surfaces, Chem. Industry Eng. Process. 33(8)(2014)2123-2129.

[22] H. Zhang, W. Li, H. Liu, D. Cui, Thermodynamic analysis on superhydrophobicity based on the design of a pillar model, Soft. Matter. 8(2012)10360-10369.

[23] M.A. Rahman, A.M. Jacobi, Wetting behavior and drainage of water droplets on microgrooved brass surface, Langmuir. 28 (2012) 13441-13451.

[24] H.Y. Zhang, Design of lotus-simulating surfaces: Thermodynamic analysis based on a new methodology, Colloid Surf. A. 413.21(2012) 314-327.

[25] Z.Y. Shi, Z.Q. Liu, H. Song, X. Zhang, Prediction of contact angle for hydrophobic surface fabricated with micro-machining based on minimum Gibbs free energy, Appl. Surf. Sci. 364(2016)597-603.

[26] G.P. Fang, Droplet motion on designed microtextured superhydrophobic surfaces with tunable wettability, Langmuir. 24.20 (2008) 11651-11660.

[27] X. Hao, G. Wu, W. Li, D. Lv, Y. Luo, L. Li, N. He, Superhydrophobic surfaces based on 
Zno-constructed hierarchical architectures, Microelectro. Eng. 141(2015)44-50.

[28] M.A. Rodríguezvalverde, M.A. CabrerizoVílchez, A new method for evaluating the most-stable contact angle using mechanical vibration, Soft. Matter. 7(1)(2010) 53-56.

[29] Y. Xiu, L. Zhu, D.W. Hess, Relationship between Work of Adhesion and Contact Angle Hysteresis on Superhydrophobic Surfaces, J. Phys. Chem.C. 112.30 (2008)11403-11407.

[30] X. Wang, X. Peng, J.F. Lu, Measuring technique of contact angle and contact angle hysteresis on rough solid surfaces I: measuring technique of contact angle, J. Basic Science and Eng. (2003)296-303. 